\title{
FUNÇÕES SPLINES APLICADAS EM DADOS DE CRESCIMENTO
}

Lorrayne Gomes ${ }^{1}$; Milena Vieira Lima; Jeferson Corrêa Ribeiro; Andreia Santos Cezário ${ }^{1}$, Eliandra Maria Bianchini Oliveira ${ }^{1}$; Wallacy Barbacena Rosa dos Santos ${ }^{1}$; Tiago Neves Pereira Valente ${ }^{2}$; Crislaine Messias de Souza ${ }^{1}$; Aline Sousa Camargos ${ }^{1}$

${ }^{1}$ Instituto Federal Goiano - IFG, Departamento de Zootecnia, Campus Morrinhos, Morrinhos, GO.

${ }^{2}$ Instituto Federal Goiano - IFG, Campus Posse. E-mail: jeferson.ribeiro@ifgoiano.edu.br

\section{RESUMO}

Em melhoramento animal, o uso de novas técnicas que permite melhorar a avaliação genética, tem sido alvo de vários estudos. Neste contexto, nos últimos anos, diversos trabalhos tiveram como objetivo, desenvolver modelos com funções mais ajustáveis às diversas variáveis. Um conjunto de funções que têm despertado atenção são as funções splines. O objetivo dessa revisão é discutir o uso das funções splines aplicadas a dados de crescimento em melhoramento animal. Splines são funções de regressão segmentada, unidas por pontos chamados de nós, com capacidade de melhorar a curvatura dos modelos, melhorando assim o ajuste de funções. Essas funções possuem propriedades interessantes sendo de natureza interpolatórias, menores problemas com multicolineriedade, linearidade nos parâmetros, além de aumentarem o domínio da aproximação, fornecendo dessa forma, estimativas num campo mais amplo de possíveis valores. Existem três tipos de funções splines: funções splines naturais, funções splines suavizantes ou regressão não paramétrica e funções splines do tipo B (B-splines). Essas últimas são as mais aplicadas em melhoramento animal, principalmente como alternativas aos modelos de regressão aleatória (MRA) que envolve o uso de polinômios de Legendre. As matrizes formadas pelos MRAs com o uso das funções B-Spline ou polinômios de Legendre são mais esparsas, sendo mais fáceis de inversão. Assim, o uso das funções splines têm sido intensificados nos últimos anos, quando o objetivo é melhorar o ajuste para análises que envolve funções, com menor número de parâmetros no modelo. Novos estudos permitirão aperfeiçoar ainda mais a técnica e descobrir novas aplicações para as funções splines.

Palavras-chave: ajuste de modelos; avaliação genética; B-spline; dados longitudinais; melhoramento animal

\section{SPLINES FUNCTIONS APPLIED IN GROWTH DATA}

\section{ABSTRACT}

In animal breeding, new methodologies can be applied in statistical analysis to improve the genetic evaluation and, for this reason, they have been the subject in several studies. In the last years, several research works have intended the model development with more adjustable functions to the distinct variables. A set of functions known as Spline functions has called the attention of researches. Then, the purpose of this review is to discuss the use of Spline functions that are applied to growth data in animal breeding. Splines are segmented regression functions that are united by points known as joint points and have the ability to improve the curvature of models and, therefore, the function adjustment. These functions have interesting properties such as the interpolatory nature, less multicolinearity problems, parameter linearity and the ability of increasing the approximation domain, all of which provide estimates in a wide range of possible values. There are three types of Spline functions: natural spline functions, smoothing spline 
functions or nonparametric regression and B-splines functions. These latter functions are more applied to animal breeding, mainly as alternatives to random regression models (RRM) that use the Legendre polynomials. The matrices formed by RRMs with the use of B-spline functions or Legendre polynomials are more scarce and easier to be inverted. Then, the use of Spline functions has been more intensified in the last years because studies have had the purpose of improving the adjustment with less model parameters in functions. New studies will allow improving the methodology and finding out new applications to the Spline functions.

Keywords: animal breeding; B-spline; genetic evaluation; longitudinal data; model adjustment

\section{INTRODUÇÃO}

No passado, para a construção de curvas suaves como o fundo de um navio ou a fuselagem de um avião, eram usadas barras longas, de material flexível que permitiam a formação de várias curvas ao longo da sua estrutura. Como auxílio, eram fixados pesos (ou nós) na porção longitudinal dessas barras, aplicando tensões capazes de manter a curvatura. O interessante desse processo é que, com o uso dos pesos ou nós, é possível flexionar as barras de forma que, a mesma, passe em pontos de interesse. Esse processo era conhecido como lofting (soteamento) e foi a base para o desenvolvimento da ideia de funções splines.

A partir da definição acima, podemos definir as funções splines como sendo a junção de vários polinôminos segmentados. Essa junção ou local onde os polinômios se unem é mais conhecida como nó (Wegman; Wright, 1983).

Para os propósitos estatísticos, splines são funções de regressão segmentadas em que se impõem restrições a fim de se unirem nos pontos chamados nós (Keele, 2008). Em suas formas mais simples, splines são modelos de regressão com um conjunto de variáveis fictícias e que se ajusta linhas de regressão separadas dentro das regiões entre os nós, e os nós ligam os ajustes de regressão segmentada. Ou seja, as funções splines são modelos com ajustes locais entre os nós que permitem estimar a forma funcional dos dados.

O rápido desenvolvimento das funções splines é devido, principalmente, a sua grande utilidade em situações aplicadas. Classes de funções splines possuem várias excelentes propriedades estruturais assim como excelentes poderes de aproximação. Como elas são fáceis de armazenar, avaliar e manipular em um computador, uma miríade de aplicações na solução numérica e uma variedade de problemas em matemática aplicada tem sido desenvolvidas (Schumaker, 2007).

Deve-se ressaltar que, conforme apresentado, até agora, as funções splines são puramente de natureza interpolatórias, e uma referência explícita ao caráter de otimização não costuma aparecer nas discussões elementares. O problema da interpolação é encaixar uma curva através de pontos $\left(x_{i}, y_{i}\right), i=1,2, \ldots, n$, no plano (Wegman; Wright, 1983). Devido ao seu caráter interpolatório, as funções splines têm sido utilizadas em diversas áreas da ciência como na engenharia, arquitetura, computação gráfica e ciências agrárias. Em zootecnia, as funções splines têm sido usadas para descrever a curva de postura em aves e curva de lactação em bovinos e caprinos. No melhoramento animal, splines têm sido empregados no ajuste da curva de crescimento dos animais e em análise de dados longitudinais através de modelos de regressão aleatória (MRA), sendo este último talvez, o que mais tenha sido favorecido através do uso das funções splines.

$\mathrm{Na}$ análise de dados longitudinais, os MRA têm sidos empregados principalmente por serem simples e com propriedades mais desejáveis (Meyer, 2005b) do que os modelos multicaracterísticos. Normalmente, os MRA utilizam os polinômios ortogonais de Legendre para a variável controle como covariáveis. Em alguns casos, são necessários polinômios de ordens mais 
altas que, por serem mais flexíveis, ajustem melhor aos dados, e têm se mostrado capazes de modelar bem mudanças nas médias e variâncias ao longo da escala contínua. Entretanto, o uso de polinômios de ordem alta gera pelo menos dois tipos de problema. O primeiro seria o "Fenômeno de Runge", que é o aumento do erro de aproximação polinomial devido ao aumento da ordem de ajuste, com erros predominantemente devido a oscilações nos extremos da curva. Os polinômios normalmente colocam grande ênfase sobre as observações nos extremos, e são conhecidos por serem potencialmente problemáticos para altas ordens de ajuste. Tal comportamento pode gerar problemas, como estimativas de componentes de variância implausíveis nos extremos da faixa da variável controle. Isso, particularmente, tem ocorrido geralmente em conjunto de dados com substancial diferença numérica de observações nos dois extremos da variável controle, e ajuste de polinômios de alta ordem (Meyer, 2005b).

Outro problema é a dificuldade para uma explicação biológica plausível para fenômenos com polinômios de altas ordens. Se fosse possível descrever um determinado fenômeno, através da interpolação dos dados, usando uma curva mais simples possível ou um conjunto de curvas, sendo essas de pequena ordem, seria mais fácil para o pesquisador explicar as variáveis que compõe tal fenômeno. As funções Splines são capazes de solucionar esses dois problemas uma vez que, em teoria, possuem melhor qualidade no ajuste, principalmente nos extremos como as BSplines (será detalhado mais adiante) e usando polinômios de pequenas ordens.

Vários trabalhos podem ser encontrados aplicando as funções splines em bovinos de leite (Bignardi et al., 2008; Bohmanova et al.,2008; Aguilar et al., 2009; Banos et al., 2009; Eaglen et al., 2011; Løvendahl et. al., 2011; Negussie et al., 2012; Steri et al., 2012; Pereira et al., 2013) em bovinos de corte (Meyer et. al., 2005a; Sánchez et al., 2008; Baldi et al., 2010, Cantet et al., 2013); em suínos (Huisman et al., 2002; Chen et al., 2011; Dufrasne et al., 2011) e em aves (Aggrey, 2002; Aggrey, 2004).

\section{FUNÇÕES SPLINES (POLINÔMIOS SEGMENTADOS)}

Funções Splines ou polinômios segmentados são pequenos segmentos de polinômios de menor grau unidos em regiões conhecidas por nós (knots). Esses nós conectam os segmentos de forma a permitir a obtenção de derivadas contínuas de grau $n-1$ (Schenkel, 1989). Os valores da função e as primeiras $n-1$ derivadas devem coincidir nos nós, portanto, a função spline é uma função contínua com $n-1$ derivadas contínuas (Paulson, 2007).

Em um sentido real, splines é uma evolução da inferência paramétrica clássica e uma ponte entre os métodos paramétricos e não paramétricos. Enquanto splines não é paramétrico na forma funcional, na maioria dos casos, podem ser escritos como uma combinação linear de funções de base que geralmente têm uma representação polinomial.

Assim, existe uma grande quantidade de classes de funções possíveis de serem utilizadas com a vantagem adicional de utilizar propriedade que permitem obter curvas mais suaves. (Wegman; Wright, 1983).

O grande obstáculo dos splines é o conhecimento dos nós. O conhecimento prévio do pesquisador na área técnica específica, o uso de diagramas e médias para a estimação grosseira de nós ou o uso de um modelo não linear para a estimação simultânea dos nós e dos coeficientes de regressão podem ser empregados nesta tarefa. O que tem sido feito na prática é a fixação dos nós por inspeção visual, ou seja, plota-se o conjunto de dados em um gráfico de dispersão, e a partir desse ponto, o pesquisador determina quais são os pontos críticos e locais onde devem inserir os nós. Para assegurar uma escolha mais correta (que minimize a soma de quadrados dos resíduos) podem-se escolher pontos próximos aos locais onde poderia estar situado o nó e então proceder a estimação dos parâmetros do modelo. $O$ ponto adotado seria aquele que minimizasse a soma de quadrados do resíduo. Segundo Schenkel (1989), este método, aparentemente trabalhoso, é exequível e bastante prático em decorrência da facilidade de computação dos polinômios. $O$ 
mesmo autor cita também as vantagens do uso das funções splines:

a) Pela inclusão de zeros nas colunas das variáveis explanatórias, há uma quebra da multicolinearidade, aumentando a confiabilidade dos valores estimados $(\hat{y})$ uma vez que minimiza o fator de inflação da variância e o viés dos estimadores.

b) O comportamento da função que descreve a relação das variáveis em uma região torna-se bastante independente do comportamento em outras regiões com características distintas, o que torna a capacidade de aderência da função spline aos pontos superiores a dos polinômios ordinários.

c) A medida de qualidade do ajustamento, dada normalmente pela soma de quadrados total devido à regressão, será bastante uniforme para a curva inteira do polinômio segmentado, independentemente da uniformidade da distribuição das observações.

d) Funções não lineares e polinômios ordinários de graus diferentes podem ser aproximados por funções splines que são lineares nos parâmetros, de fácil estimação, com propriedades ótimas (BLUE) e de simples interpretação biológica.

e) Aumentam o domínio da aproximação, isto é, fornecem estimativas num campo mais amplo de possíveis valores.

Um exemplo que define bem a vantagem do uso de splines em detrimento a outros polinômios, principalmente os polinômios de Legendre, podem ser analisados nos trabalhos de Bohmanova et al., (2008), ilustrado na figura 1. Nesse trabalho, um polinômio de Legendre de ordem quatro foi comparado com três polinômios segmentados (spline) contendo quatro, cinco ou seis nós, em modelos de regressão aleatória, aplicados à característica de escore de células somáticas em bovinos de leite na raça holandesa (figura 1). Na figura, a variância genética aditiva para produção de leite foi plotada, considerando três lactações. Nesse trabalho, os autores apontam os melhores resultados aos obtidos com o uso de splines com seis nós (a função spline que continha seis nós teve melhor desempenho geral, com base nos critérios considerados). Mas é possível observar que a as curvas usando spline (quatro, cinco ou seis nós) se ajustaram melhor aos dados do que os polinômios de Legendre. Na literatura, é possível encontrar vários trabalhos comparando a qualidade de ajuste das funções splines em relação a outros polinômios.

\section{FUNÇÕES SPLINES NATURAIS}

Splines cúbicos são as funções spline mais populares. Elas são funções suaves com os quais se encaixam os dados, e quando usados para a interpolação, não têm o comportamento oscilatório, que é característica de uma interpolação polinomial de alto grau (como interpolação de Lagrange, interpolação de Hermite, etc). Keele (2008) cita que uma limitação das funções splines cúbicas (isso pode ser estendido a funções de outros graus) é que as funções segmentadas são ajustadas apenas entre cada nó. Para dados que caem antes do primeiro nó e além do último nó, não se ajusta uma spline. Sem ajustes para os limites dos dados, é possível que o ajuste devido à função spline se comporte erraticamente em torno dos limites de $x$. Funções splines cúbicas naturais adicionam dois nós para ajustar nos valores mínimos e máximos de $x$ e ajustam uma função linear entre os nós adicionais no limite e os nós interiores. Isto força o ajuste devido à função spline ser linear antes do primeiro nó e depois do último nó. Tal linearidade forçada nos limites evita comportamento "selvagem" no ajuste dos dados perto dos extremos dos dados. Como pouco é perdido ao se usar funções splines cúbicas naturais enquanto alguns ganhos no ajuste do modelo são possíveis, normalmente estas são preferidas em relação às funções splines cúbicas.

Basicamente, existem três tipos de funções splines: funções splines naturais, funções splines do tipo B e funções splines suavizantes ou regressão não paramétrica. Vamos definir cada uma dessas funções a seguir. 


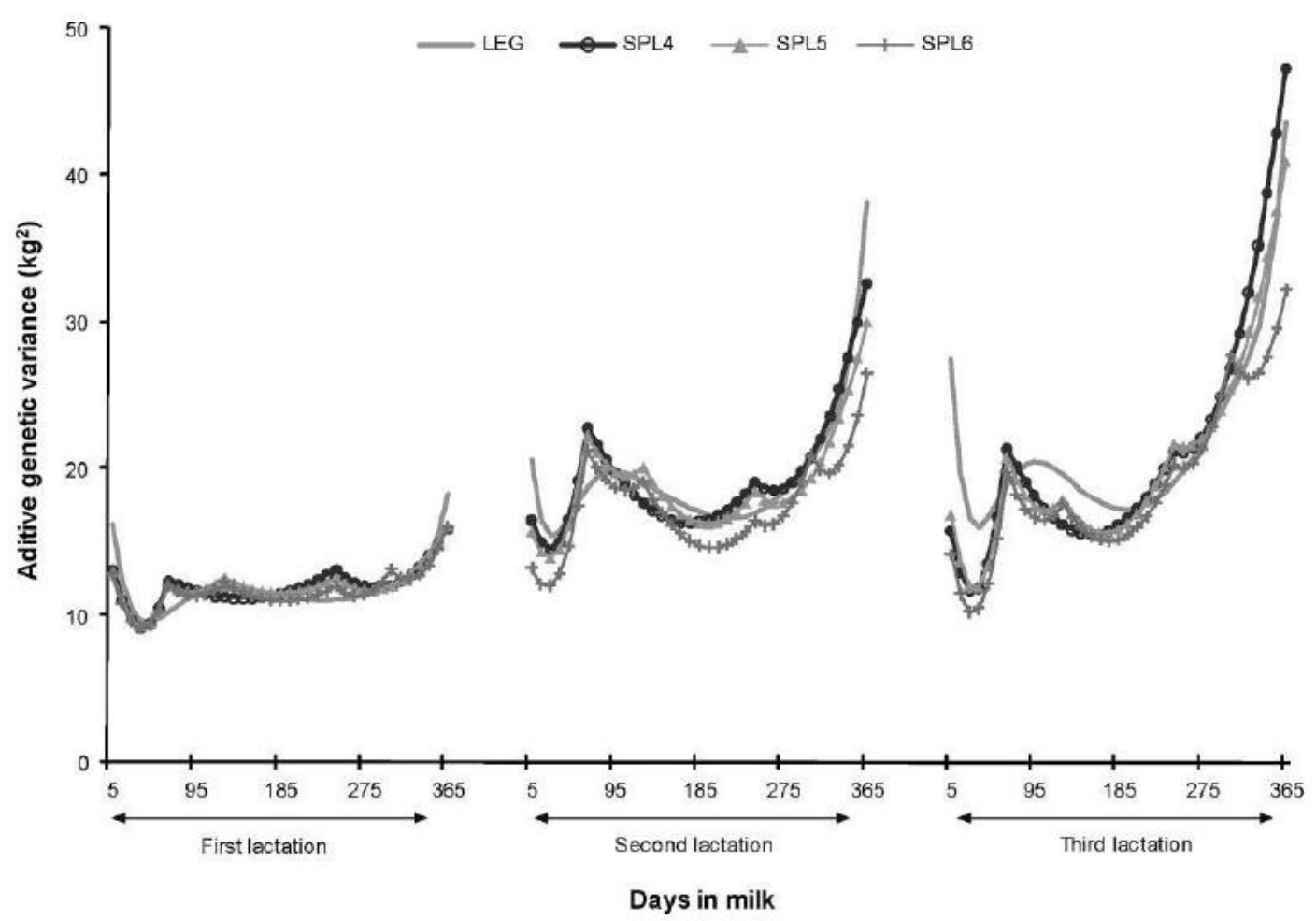

Figura 1. Adaptado de Bohmanova et al., (2008). Média posteriori de estimativas de variância genética aditiva de produção de leite diária considerando a primeira, secunda e terceira lactação (LEG = modelo com Polinômios de Legendre; SPL4, SPL5 e SPL6 = modelo com splines linear com 4, 5 ou 6 nós, respectivamente).

\section{FUNÇÕES SPLINES DO TIPO B}

Em casos específicos, nas funções splines cúbicas (naturais ou outras), as colunas de $\mathrm{X}$, na matriz do modelo, tende a ser altamente correlacionada uma vez que cada coluna é uma versão transformada de $x$, o que pode induzir a considerável colinearidade. Essa colinearidade pode resultar em uma matriz quase singular, o que causa uma imprecisão no ajuste do spline (Keele, 2008).

Para resolver esse problema, a escolha comum são funções B-spline, que geram resultados de ajustes equivalentes às funções splines já mencionadas, mas têm melhores propriedades numéricas (Rupert et al., 2003). Aqui, o "B" significa base (de Boor, 2001). Meyer (2005b) cita que as funções B-Splines compreendem um conjunto de funções sobrepostas, suaves e não negativa, que são unimodal e acrescenta uma unidade em todos os valores de $t$, podendo-se ter funções Bsplines de diferentes graus.

Tal como splines com base na forma truncada, as funções B-splines podem ter diferentes graus de $p$, além de poder ser definida de forma recursiva. As funções de base de grau $p=0$ têm valores de unidade para todos os pontos em um determinado intervalo, e zero caso contrário.

Para $o k^{\text {ésimo }}$ intervalo dado pelos nós $T_{k}$ e $T_{k+1} \operatorname{com} T_{k} \leq T_{k+1}$, temos:

$$
B_{k, 0}(t)=\left\{\begin{array}{l}
1, \text { se } T_{k} \leq t \leq T_{k+1} \\
0, \text { caso contrário }
\end{array}\right.
$$

Funções base de graus mais altos, $B_{k, p}$ para $p>0$, são então determinados a partir dos valores de funções base de grau menor, já calculadas, e da largura dos intervalos adjacentes entre nós. A relação geral, de acordo com de Boor (2001), é: 


$$
B_{k, p}(t)=\frac{t-T_{k}}{T_{k+p}-T_{k}} B_{k, p-1}(t)+\frac{T_{k+p+1}-t}{T_{k+p+1}-T_{k+1}} B_{k+1, p-1}(t)
$$

Para cada valor de $p$, há um número limitado de funções de base não nulas de menor grau, que podem ser explorados, ao se calcular a equação anterior. Estratégias eficientes são descritas em de Boor (2001). Alternativamente, para nós igualmente espaçados, funções B-splines podem ser obtidas como diferenças entre funções splines com uma base de funções de grau truncado.

Para uma dada faixa de $t, T_{0}$ a $T_{m}$, divisões em $m$ intervalos requer a especificação de $m-1$ nós internos. Junto com os dois nós externos $\left(T_{0}\right.$ e $\left.T_{m}\right)$, isto gera $m+1$ pontos de nó e $m+p$ funções não nulas $B_{k, p}$ a serem consideradas. O cálculo da equação anteriormente definida, entretanto, requer $p$ adicionais nós a serem especificados em cada lado do intervalo. Assim, tem-se $2 p+m+1$ nós no total. Para funções B-splines "uniformes" com nós colocados em intervalos iguais $\Delta$, Eilers e Marx (2010) sugerem colocar os nós adicionais em intervalos iguais fora da faixa de $t$, isto é, em $T_{0}-p \Delta, \ldots, T_{0}-\Delta e T_{m}+\Delta, \ldots, T_{m}+p \Delta$. Isso resultaria em todos as $B_{k, p}$ para um dado $p$ terem a mesma forma, ou seja, simplesmente serem cópias horizontalmente deslocadas uma da outra. Alternativamente, se poderiam definir os dois nós externos tendo multiplicidade $p+1$, isto é, colocar os $p$ nós adicionais de ambos os lados do valor correspondente ao nó externo. Isto é prática comum na implementação de B-splines em pacotes estatísticos, como o R (Meyer, 2005a). Para $p \geq 2$, isto proporcionaria $B_{k, p}$ de diferentes formas para aqueles envolvendo somente nós internos e aqueles envolvendo nós externos e adicionais, mesmo se todos os nós internos são espaçados em intervalos iguais.

Como as splines com base truncada, as funções B-splines podem ser penalizadas para obter curvas mais suaves. Essa penalidade resultou em uma formulação análoga a uma regressão de cumeeira. Penalidades correspondentes para uma base B-spline, sugeridas por Eilers e Marx (1996), são diferentes com base onde o grau de ajuste e a ordem de diferença para a pena pode ser escolhido de forma independente. Eilers e Marx (1996) refere-se às funções que resultam como "P-splines", enquanto que outros autores usam o termo P-splines para quaisquer splines penalizados, independentemente da base. A análise detalhada das propriedades de B-splines e Psplines são dadas por Eilers e Marx (2010).

\section{FUNÇÕES SPLINES SUAVIZANTES OU REGRESSÃO NÃO PARAMÉTRICA}

Em alguns casos, pode acontecer que a curva associada à função spline tenha um aspecto ondulado. Isso acontece quando o número de nós é grande, com vários polinômios de pequena ordem, situação típica quando há excesso no número de parâmetros. Essa facilidade em exceder o número de parâmetros, tem gerado críticas entre os pesquisadores, devido a grande flexibilidade das funções splines, causando superajuste dos dados (overfit).

Críticos argumentam que enquanto estimadores não paramétricos não lineares fazem poucas pressuposições, eles também facilmente revelam variações locais estranhas entre $x$ e $y$ que não são de interesse. Em resumo, em vez de observar uma estimativa da provável forma funcional não linear, o analista observa uma estimativa não linear devido ao superajuste.

Modelos muito parametrizados podem ocorrer tanto em regressão paramétricas quanto em regressão não paramétrica. Entretanto, não se pode reduzir o número de parâmetros aleatoriamente. Assim, o que pode ser feito para tal redução é o uso de algum critério, como o AIC, podendo assim, serem aplicados às funções splines (Keele, 2008). Estes problemas podem ser atenuados também através da imposição de uma pena, o que reduz a influência dos coeficientes de regressão, com o intuito de produzir uma curva mais suave (Meyer, 2005b). O uso de penalizações em funções splines foi proposto por Eilers e Marx (1996), a partir de funções splines do tipo B (próxima secção) com o intuito de a penalidade oferecer um caráter contínuo, adicional 
e controlar a suavidade da curva (Eilers; Marx, 2010). Rupper et al., (2003) sugeriu que essa penalização poderia ocorrer na soma de quadrados dos coeficientes da regressão na equação, ou seja, minimizando a soma de quadrados entre $y$ e a estimativa não paramétrica $f(x)$. Como os modelos com funções splines são estimados por quadrados mínimos, eles compartilham as propriedades de modelos de regressão linear. Isso implica que uma estimativa spline $f$, minimiza a soma de quadrados entre $y$ e a estimativa não paramétrica $f\left(x_{i}\right)$, ou seja:

$$
S Q(f)=\sum_{i=1}^{n}[y-f(x)]^{2}
$$

Entretanto, a estimativa de $f$ que minimize a equação acima pode usar muitos parâmetros. A solução da estimação penalizada é anexar uma penalidade para o número de parâmetros usados para estimar $f$. Isso sugere que se minimiza $S Q(f)$, mas sujeito a um termo de restrição ou penalidade para o número de parâmetros locais usados. A penalidade usada para modelos splines é:

$$
\lambda \int_{X_{1}}^{X_{n}}\left[f^{\prime \prime}(x)\right]^{2} d x
$$

que implica que a estimação spline é:

$$
S Q(f, \lambda)=\sum_{i=1}^{n}[y-f(x)]^{2}+\lambda \int_{1}^{X_{n}}\left[f^{\prime \prime}(x)\right]^{2} d x
$$

Minimizando a soma de quadrados entre $y$ e a estimativa não paramétrica, $f(x)$ sujeita à penalidade na equação anterior.

A restrição usada anteriormente é conhecida como uma penalidade de rugosidade. Esta penalidade tem duas partes.

A primeira parte é $\lambda$, normalmente chamada de parâmetro suavizante, e a segunda, é a integral do quadrado da derivada segunda de $f(x)$. A lógica por trás do uso da integral do quadrado da derivada segunda de $f(x)$ é bastante intuitiva. A segunda derivada mede a taxa de mudança da inclinação de uma função ou curvatura. Um alto valor para a segunda derivada significa alta curvatura e vice-versa. Através do uso da integral do quadrado, o termo gera uma medida da curvatura ao longo de toda a faixa da estimativa não paramétrica. Quando esta é alta, $f(x)$ é mais rugosa, e quando é pequena, $f(x)$ é mais suave.

0 parâmetro $\lambda$, o qual é não negativo, diretamente controla a quantidade de peso dada à medida da segunda derivada de quão suave $f$ é. Portanto, $\lambda$ estabelece uma relação de troca entre proximidade de ajuste nos dados e a penalidade. À medida que o valor de $\lambda$ diminui, $f(x)$ interpola os dados, e tem-se um ajuste rugoso. Por outro lado, à medida que $\lambda \rightarrow \infty$ a segunda derivada integrada é restringida a ser zero, e o resultado é um ajuste global de quadrados mínimos mais suaves. De forma mais geral, quando $\lambda$ cresce, tem-se um ajuste mais suave aos dados devido talvez ao ajuste viesado, e quando o parâmetro decresce, o ajuste revela menos viés, mas com variância aumentada.

Segundo Green e Silverman (1994), há duas abordagens diferentes para a questão da escolha do parâmetro suavizante $\lambda$. A primeira abordagem é considerar a livre escolha do parâmetro suavizante como um atributo vantajoso do procedimento. Variando $\lambda$, os atributos dos dados que surgirem em diferentes escalas podem ser explorados e, se uma estimativa única é necessária, esta pode ser obtida por uma escolha subjetiva. Segundo estes autores, há uma possibilidade que tal abordagem subjetiva seja, na realidade, a mais útil.

A outra abordagem tem uma visão filosófica oposta à primeira. É necessário um método 
automático em que a escolha do valor do parâmetro suavizante é feita pelos dados, ou seja, que a escolha seja objetiva. Green e Silverman (1994) afirmam que há diversos métodos automáticos disponíveis, sendo que provavelmente o mais bem conhecido seja o método de validação cruzada.

\section{ALGUMAS APLICAÇÕES DE FUNÇÕES SPLINES NO MELHORAMENTO ANIMAL}

No melhoramento animal, uma das grandes utilizações das funções splines estão nas análises de regressão aleatória. Os MRAs, quando aplicados aos dados de animais, têm sido realizados principalmente com o uso de funções polinomiais ajustadas, ou seja, com poucos coeficientes de regressão (Meyer, 2005b). Uma alternativa lógica em P-Splines, como descrito acima, é encaixar uma regressão aleatória com um número limitado de funções de base B-spline, assumindo que a matriz de covariância dos coeficientes da regressão aleatória não seja estruturada, tal como descrito por Rice e Wu (2001). A mesma abordagem foi feita pelo Shi et al. (1996), e James et al. (2000), que combinando também a redução de estimativa de classificação para evitar o excesso de parametrização.

Os modelos de regressão aleatória são mais aplicados a dados longitudinais, ou seja, medidas tomadas ao longo do tempo como dados de crescimento, postura ou lactação. Os modelos de repetibilidade fornecem predições únicas, válidas para todas as idades e ambientes considerados. Os modelos multicaracterísticos resultam em predições para número limitado de idades ou ambientes. Os MRAs permitem a predição de coeficientes de regressão que representam o comportamento do valor genético aditivo de cada animal na característica avaliada em função do tempo (idade) ou qualquer outra variável contínua. Desta forma, infinitos valores da variável independente são considerados dentro de um intervalo definido. Por este motivo, o modelo não exige utilização de fatores de ajustes para idades consideradas como padrão ou projeções de lactações parciais para produção total e permite uma melhor utilização dos dados disponíveis, já que todas as informações do animal e de seus parentes são utilizadas (Silva et al., 2008). Outra vantagem é que as matrizes formadas pelos MRAs com o uso das funções B-Spline ou polinômios de Legendre são mais esparsas, sendo mais fáceis de inversão. Para uma função BSpline de grau $p$, cada linha da matriz terá $p+1$ coeficientes, e as exigências computacionais são menores, do que análises correspondentes com o mesmo número de coeficientes para uma base polinomial.

Veremos agora, alguns exemplos de aplicações de modelos de regressão aleatória com o uso de funções Splines em espécies de interesse zootécnico.

\section{BOVINOS DE CORTE}

Meyer (2005a) trabalhando com bovinos da raça Angus Australiano avaliou a curva de crescimento utilizando funções B-splines em MRA como uma alternativa aos polinômios ortogonais de Legendre. $O$ banco de dados usado continha 84.533 registros de 20.731 animais pertencentes 43 rebanhos, com alta proporção dos animais com quatro ou mais pesos registrados. Para este trabalho foram usados dados de pesos do nascimento aos 820 dias de vida. Um total de treze análises, considerando diferentes combinações de funções B-splines linear, quadrática e cúbica e até seis nós, foram realizadas. No geral, análises usando B-splines mostraram-se mais robustas contra os problemas de ajuste nos extremos dos dados, comuns em análises com polinômios ortogonais. Segundo a autora, um MRA com uma função B-spline quadrática, com nós nos dias 0, 200, 400, 600 e 821 e um total de 91 componentes de variância parece ser a melhor opção, pois oferece equilíbrio entre detalhamento do modelo, número de parâmetros a ser estimada, plausibilidade dos resultados e qualidade de ajuste, medido como o quadrado médio do resíduo.

Sánchez et al. (2008) realizaram estudo a fim de examinar a praticabilidade de se usar MRA com função spline linear no ajuste de características de crescimento em uma população 
multirracial de bovinos de corte. Para atingir o objetivo, os resultados foram comparados com aqueles obtidos com um modelo multicaracterístico tradicional, quando ambos os modelos foram aplicados a um banco de dados com 1.800 .000 de registros de 1.100 .000 de animais pertencentes à Associação Americana de Gelbvieh. Os autores concluíram que, o MRA com função spline linear por evitar o descarte de registros que estão fora das usuais faixas de mensurações, permite que maiores acurácias sejam alcançadas e, portanto maior progresso genético poderia ser esperado.

Em outro trabalho, realizado por Baldi et al., (2010) com bovinos da raça Canchim, o objetivo era estimar funções de covariância utilizando modelos de regressão aleatória comparando polinômios de Legendre e funções B-Splines da idade do animal, para pesos do nascimento à idade adulta. Foram comparados também os resultados obtidos por MRA e modelos multicaracterísticos. Os dados para esse estudo continha 49.011 registros em 2.435 fêmeas. 0 modelo de análise incluiu os efeitos fixos de grupo contemporâneo, idade da mãe como covariável e tendência quadrática média da população. Um total de dezessete análises foi utilizado, considerando efeitos quadrático e cúbico, contendo até sete nós. Segundo a conclusão do autor, o modelo de ajuste quadrático B-Splines que continha quatro nós ou três segmentos para o efeito genético aditivo direto e efeito ambiental permanente e dois nós para o efeito genético aditivo materno e de ambiente permanente materno, foi o mais adequado para descrever a estrutura de covariância dos dados. Os modelos de regressão aleatória usando as funções B-spline como funções básicas se ajustou aos dados melhores do que polinômios de Legendre, especialmente na idade adulta, entretanto um número maior de parâmetros precisa ser estimado ao usar funções BSplines.

\section{BOVINOS DE LEITE}

Bohmanova et al., (2008) estudando dados de produção de leite, gordura e proteína no dia do controle e também de escores de células somáticas de vacas holandesas no Canadá, compararam 4 modelos de regressão aleatória, em que as regressões fixas e aleatórias eram ambas ajustadas por polinômios de Legendre de ordem 4 ou funções splines lineares com 4, 5 ou 6 nós. Foram consideradas 12 classes de variância residual. Os autores concluíram que o modelo que utilizava funções splines lineares com 6 nós foi superior aos demais modelos, devendo ser preferido para as avaliações genéticas da raça no Canadá.

Bignardi et al., (2008) estudaram funções B-splines para ajuste de dados de produção de leite no dia do controle de primeiras lactações de vacas da raça Holandesa no Brasil. Os efeitos aleatórios genético-aditivo e de ambiente permanente foram modelados por meio de funções Bsplines lineares, quadráticas ou cúbicas, com até 7 nós. $O$ resíduo foi considerado heterogêneo, contendo 6 classes de variâncias. O modelo empregando uma B-spline cúbica com o número de coeficientes de regressão aleatória igual 8 tanto para o efeito aleatório genético aditivo como de ambiente permanente foi o mais adequado para ajustar os dados. Para tal modelo, os valores de herdabilidade variaram de 0,20 e 0,40 ao longo da lactação.

Um estudo com dados de primeira lactação de vacas da raça Ayrshire para a análise genética de mastite clínica foi conduzido por Negussie et al., (2012). Para esse estudo, o banco de dados era composto por 112.000 registros. Splines com quatro e seis nós foram usadas em comparação com polinômios de Legendre de terceira ordem, para modelar a trajetória genética da mastite clínica. Componentes de covariância foram estimadas utilizando modelos bayesianos de limiar e procedimentos Monte Carlo via cadeias de Markov (MCMC). Os autores afirmaram que as estimativas obtidas por polinômios de Legendre foram particularmente elevadas e errôneas em ambas as extremidades da trajetória da lactação, enquanto as estimativas obtidas via função spline, não tiveram problemas de ajuste. Além disso, tanto as splines de quatro nós quanto as de seis nós tiveram excelente ajuste com uma correlação entre eles de 0,96, com leve superioridade da função que continha quatro nós. 
Pereira et al. (2013) analisou dados de 59.744 dados de produção diária de leite de 7.810 primeiras lactações de fêmeas da raça Gir e Girolando. Os efeitos aditivos genéticos e de ambiente permanente foram modelados utilizando polinômios ortogonais de Legendre ortogonais ou Splines lineares. As variâncias residuais foram modeladas considerando 1, 5 ou 10 classes de dias em lactação. $O$ modelo que melhor ajustou os dados foram os que continham splines com seis nós, para a variância residual. Entretanto, um modelo usando um polinômio de terceira ordem para o efeito genético aditivo e polinômio de quarta ordem para os efeitos de ambiente permanente fora os que mais se ajustaram aos dados. Uma explicação para que as funções splines não tenham ajustado bem aos dados pode estar na escolha dos nós. Os autores propuseram novos estudos considerando outros nós, na esperança que o ajuste seja mais parcimonioso.

\section{SUÍNOS}

Huisman et al., (2002) realizaram estudo a fim de investigar qual tipo de MRA melhor ajusta dados de peso de suínos. Dois MRA que descreveram o peso individual de suínos, um usando polinômio ortogonal de Legendre cúbico e outro usando função spline cúbica natural, foram comparados. Estes dois modelos são foram comparados com um modelo multicaracterístico. Ambos os MRA apresentaram melhor ajuste do que o modelo multicaracterístico, no entanto, os autores não chegaram à conclusão de qual MRA foi melhor, pois MRA com polinômio ortogonal de Legendre foi superior pelo BIC, enquanto o MRA com função spline cúbica natural foi superior pelo AIC.

Com o intuito de estudar as estimativas de parâmetros genéticos para o número de natimortos em relação ao tamanho da leitegada por meio de MRAs, Chen et al., (2011), utilizaram dados de 6.575 animais de primeira leitegada e 6.259 de segunda leitegada, a partir de 4 linhagens de suínos da raça Duroc. Foram utilizados polinômios de Legendre e Splines para modelagem dos efeitos aleatórios. Para os dados da primeira leitegada, a ordem polinomial, o número de nós, e o número de intervalos utilizados para os respectivos modelos, foram quadrático, cúbico e cúbico respectivamente, com o uso de funções B-splines lineares. Para os modelos quem foram aplicados aos dados de segunda leitegada, foram utilizados polinômios, onde o número de nós e o número de intervalos utilizados para os respectivos modelos eram quadrático, cúbico e cúbico respectivamente. Tanto na primeira lactação quanto na segunda lactação, as curvas ajustada por splines tiveram melhores resultados, entretanto, foi observado a presença de artefatos nos modelos, devido aos dados não terem ajustado bem aos polinômios aplicados nesse estudo.

Em outro estudo, Dufrasne et al., (2011) analisaram dados de crescimentos de leitões filhos do cruzamento de reprodutores da raça Piétrain acasalados com fêmeas da raça Landrace. Os dados eram compostos por 12.610 registros de crescimentos coletadas em 1.435 suínos híbridos de machos Piétrain e porcas Landrace, em estações de teste e 52.993 registros de crescimentos coletados em 50.670 suínos, com a composição racial de pelo menos $40 \%$ Piétrain ou Landrace, em teste na fazenda. Os dados foram analisados por meio de modelos de regressão aleatória bivariada, com o uso de spline lineares, sendo que, para os dados originados nas estações de teste foram utilizados quatro nós (50, 100, 175 e 210 dias de vida), enquanto nos teste em fazenda foram utilizados três nós (175, 210 e 250 dias de vida). Segundo os autores o modelo testado ajustou-se bem aos dados com coeficiente de determinação variando de 0,60 a 0,99, dependendo da idade considerada.

\section{AVES}

Arango et al., (2009) usaram modelos de regressão aleatória para explorar a trajetória peso do ovo. Nesse estudo, os autores utilizaram dados de duas populações da linhagem Hy-line brancas (WL) e marrons (BL) contendo 196.778 e 94.768 observações acumuladas através de quatro gerações de 25.257 e 17.276 indivíduos. Os coeficientes de spline linear em cada ponto de 
idade (d) foram calculados utilizando-se três nós (K) que representaram pontos de flexão da trajetória, e as idades correspondentes para RAM. Após análises preliminares, ficaram definidos os nós nos nas idades de 140 (K1), 185 (K2) e 295 (K3) dias. As estimativas de herdabilidade e repetibilidade em cada nó foram 0,32, 0,42 e 0,56 e 0,66, 0,65 e 0,72 para WL. Valores correspondentes foram de 0,45, 0,55 e 0,54, e 0,71, 0,71 e 0,77 para BL. As correlações genéticas foram relativamente altas entre $\mathrm{K} 2$ e $\mathrm{K} 3(0,74$, para $\mathrm{WL}$ e 0,77 para $\mathrm{BL})$, mas moderada entre $\mathrm{K} 1$ e K2 ou K3 $(0,33-0,57)$. Os autores concluíram que os modelos contendo spline foram eficientes em estimar os parâmetros genéticos no estudo.

\section{CONSIDERAÇÕES FINAIS}

O uso das funções splines têm sido intensificados nos últimos anos, quando o objetivo é melhorar o ajuste para análises que envolve funções, com menor número de parâmetros no modelo. As funções B-splines são as mais utilizadas em melhoramento animal, principalmente em modelos de regressão aleatória, em substituição aos polinômios de Legendre, resultando em funções mais esparsas e mais fáceis para obter a matriz inversa, demandando de menor esforço computacional. Novos estudos permitirão aperfeiçoar ainda mais a técnica e descobrir novas aplicações para as funções splines.

\section{REFERÊNCIAS}

AGGREY, S.E. Comparison of Three Nonlinear and Spline Regression Models for Describing Chicken Growth Curves. Poultry Science, v.81, p.1782-1788, 2002. https://doi.org/10.1093/ps/81.12.1782

AGGREY, S.E. Modelling the effect of nutritional status on pre-asymptotic andrelative growth rates in a random-bred chicken population. Journal of Animal Breeding and Genetics, v.121, p.260-268, 2004. https://doi.org/10.1111/j.1439-0388.2004.00462.x

AGUILAR, I.; MISZTAL; I.; TSURUTA, S. Genetic components of heat stress for dairy cattle with multiple lactations. Journal of Dairy Science, v.92, p.5702-5711, 2009. https://doi.org/10.3168/jds.2008-1928

ARANGO, J.A.; TURKMUT, L. SETTAR, P.; O'SULLIVAN, N.P. A random regression approach to model egg weight trajectory in two layer populations. In: 6th European Poultry Genetic Symposium, 2009, Bedlewo near Poznan. Proceedings... Bedlewo near Poznan, 2009.

BALDI, F.; ALENCAR, M.M.; ALBUQUERQUE, L.G. Random regression analyses using B-splines functions to model growth from birth to adult age in Canchim cattle. Journal of Animal Breeding and Genetics, v.127, p.433-441, 2010. https://doi.org/10.1111/j.1439-0388.2010.00873.x

BANOS, G.; COFFEY, M.P. Genetic association between body energy measured throughout lactation and fertility in dairy cattle. Animal, v.4, n.2, p.189-199, 2010. https://doi.org/10.1017/S1751731109991182

BIGNARDI, A.B.; EL FARO, L.; ALBUQUERQUE, L.G.; TORRE JÚNIOR, R.A.A. Modelos de regressão aleatória empregando funções B-splines para modelar a produção de leite de vacas da raça Holandesa. In: Reunião Anual da Sociedade Brasileira De Zootecnia, 45, 2008, Lavras. Anais... Lavras: SBZ, 2008. (CD-ROM).

BOHMANOVA, J.; MIGLIOR, F.; JAMROZIK, J.; MISZTAL I.; SULLIVAN P.G. Comparison of random regression models with Legendre polynomials and linear splines for production traits and somatic 
cell score of Canadian Holstein cows. Journal of Dairy Science, v.91, p.3627-3638, 2008. https://doi.org/10.3168/jds.2007-0945

de BOOR C, A Practical Guide to Splines, New York: Springer Verlag, 2001.

DUFRASNE, M.; RUSTIN, M.; JASPART, V.; WAVREILE, J.; GENGLER, N. Using test station and onfarm data for the genetic evaluation of Piétrain boars used on Landrace sows for growth performance. Journal of Animal Science, v.89, p.3872-3880, 2011. https://doi.org/10.2527/jas.2010-3816

CANTET, R.J.C.; BIRCHMEIER, A.N.; CANAZA CAYO, A.W.; FIORETTI, C. Semiparametric animal models via penalized splines as alternatives to models with contemporary groups. Journal of Animal Science, v.83, p.2482-2494, 2005. https://doi.org/10.2527/jas.2010-3816

CHEN, C.Y.; MISZTAL, I.; TSURUTA, S.; HERRING, W.O.; HOLL, J.; CULBERTSON M. Genetic analyses of stillbirth in relation to litter size using random regression models. Journal of Animal Science, v.88, p.3800-3808, 2010. https://doi.org/10.2527/jas.2009-2413

EAGLEN, S.A.E.; COFFEY, M.P.; WOOLLIAMS, J.A.; MRODE, R.; WALL, E. Phenotypic effects of calving ease on the subsequent fertility and milk production of dam and calf in UK HolsteinFriesian heifers. Journal of Dairy Science, v.94, p.5413-5423, 2011. https://doi.org/10.3168/jds.2010-4040

EILERS, P.H.C; MARX, B.D. Flexible smoothing with B-splines and penalties. Statistical Science, v.11, p.89-121, 1996. https://doi.org/10.1214/ss/1038425655

EILERS, P.H.C.; MARX, B.D. Splines, knots, and penalties. WIREs Computational Statistics, v.2, n.6, p.637-653, 2010. https://doi.org/10.1002/wics.125

GREEN, P.J.; SILVERMAN, B.W. Nonparametric regression and Generalized Linear Models. A Roughness Penalty Approach. London: Chapman \& Hall / CRC, 1994. https://doi.org/10.1007/978$\underline{1-4899-4473-3}$

HUISMAN, A.E.; VEERKAMP, R.F.; VAN ARENDONK, J.A. Genetic parameters for various random regression models to describe the weight data of pigs. Journal of Animal Science, v.80, p.575-582, 2002. https://doi.org/10.2527/2002.803575x

JAMES, G.M.; HASTIE T.J, SUGAR C.A. Principal component models for sparse functional data. Biometrika, v.87, p.587-602, 2000. https://doi.org/10.1093/biomet/87.3.587

KEELE, L.J. Semiparametric regression for the social sciences. San Francisco: John Wiley and Sons, 2008.

LØVENDAHL, P.; CHAGUNDA, M.G.G. Covariance among milking frequency, milk yield, and milk composition from automatically milked cows. Journal of Dairy Science, v.94, p.5381-5392, 2011. https://doi.org/10.1093/biomet/87.3.587

MEYER, K. Random regression analyses using B-splines to model growth of Australian Angus cattle. Genetic Selection Evolution, v.37, p.473-500, 2005a. https://doi.org/10.1051/gse:2005012 
MEYER, K. Advances in methodology for random regression analyses. Australian Journal of Experimental Agriculture, v.45, p.847-858, 2005b. https://doi.org/10.1071/EA05040

NEGUSSIE, E.; STRANDÉN, I.; TSURUTA, S.; MANTYSAARI, E.A. Longitudinal threshold model analysis of clinical mastitis using linear splines. Livestock Science, v.149, p.173-179, 2012. https://doi.org/10.1016/i.livsci.2012.05.019

PAULSON, D.S. Handbook of regression and modeling - Application for the clinical and pharmaceutical industries. New York: Chapman \& Hall / CRC, 2007.

PEREIRA, R.J.; BIGNARDI, A.B.; EL FARO, L.; VERNEQUE, R.S.; VERCESI FILHO, A.E.; ALBUQUERQUE, L.G. Random regression models using Legendre polynomials or linear splines for test-day milk yield of dairy Gyr (Bos indicus) cattle. Journal of Dairy Science, v.96, p.565-574, 2013. https://doi.org/10.3168/ids.2011-5051

SÁNCHEZ, J.P.; MISZTAL, I.; AGUILAR, I.; BERTRAND, J.K. Genetic evaluation of growth in a multibreed beef cattle population using random regression-linear spline models. Journal of Animal Science, v.86, p.267-277, 2008. https://doi.org/10.2527/jas.2007-0064

SCHENKEL F.S. Utilização de polinômios segmentados na pesquisa zootécnica: considerações teóricas e práticas [notas]. Porto Alegre (RS):UFRGS; 1989

SCHUMAKER, L.L, Spline functions: basic theory. Tenessee: Cambrigde Mathematical Library, 2007. https://doi.org/10.1017/CBO9780511618994

SILVA, M.A, THIÉBAUT, J.T.L., VALENTE, B.D., TORRES, R.A., FARIA, F.J.C. Modelos Lineares Aplicados ao Melhoramento Genético Animal. Belo Horizonte: FEPMVZ Editora, 2008. p.322-332.

SHI, M.; WEISS R.E.; TAYLOR, J.M.G. An analysis of pediatric CD4 counts for acquired immune deficiency syndrome using flexible random curves. Journal of the Royal Statistical Society, v.45, p.151-164, 1996. https://doi.org/10.2307/2986151

WEGMAN, E.J.; WRIGHT, I.W. Splines in statistics. Journal of the American Statistical Association v.78, n.382, p.351-365, 1983. https://doi.org/10.2307/2288640 